\title{
ASI-3: NUEVA ESCALA PARA LA EVALUACIÓN DE LA SENSIBILIDAD A LA ANSIEDAD
}

\author{
Bonifacio Sandin, Rosa M. Valiente, Paloma Chorot y Miguel A. Santed
}

Facultad de Psicología, Universidad Nacional de Educación a Distancia, Madrid

\begin{abstract}
Resumen: La Anxiety Sensitivity Index-3 (ASI-3) es una nueva escala de autoinforme de 18 items diseñada para la evaluación de las tres facetas más replicadas de la sensibilidad a la ansiedad, i.e., las dimensiones física, cognitiva y social (Taylor et al., 2007). En la presente investigación se ha estudiado la estructura factorial, la fiabilidad, la validez y los datos normativos de la versión española de la ASI-3 en una muestra de estudiantes universitarios $(\mathrm{N}=582)$. Mediante la aplicación de análisis factoriales exploratorios y confirmatorios se demuestra la existencia de una estructura jerárquica, consistente en tres factores primarios y un factor de orden superior, siendo los tres factores primarios idénticos a los descritos por Taylor et al. (2007). Los datos sobre fiabilidad (consistencia interna y test-retest) y validez (convergente y discriminante) sugieren que la ASI-3 posee excelentes propiedades psicométricas. Los resultados sobre datos normativos indican que las mujeres puntúan más alto que los varones en la subescala física de la ASI-3 y en la ASI-3 total. Se discuten las implicaciones de estos resultados sobre la psicopatología y la psicología clínica.
\end{abstract}

Palabras clave: ASI-3, sensibilidad a la ansiedad, trastornos de ansiedad, psicopatología, evaluación, análisis factorial confirmatorio, EQS, PANAS, SA-45, SCL-90.

\section{ASI-3: A new scale for the assessment of anxiety sensitivity}

\begin{abstract}
The Anxiety Sensitivity Index-3 (ASI-3) is a new 18-item self-report multidimensional instrument designed to assess the three best replicated dimensions of anxiety sensitivity, i.e., Physical, Cognitive and Social Concerns (Taylor et al., 2007). In the present study we examined the factor structure, reliability, validity and normative data of the Spanish version of the ASI-R in a nonclinical sample of 582 undergraduates. Exploratory and confirmatory factor analyses indicate a hierarchical factor structure consisting of 3 lower-order factors and 1 higher-order factor; where the 3 primary factors were identical to the ones reported by Taylor et al. (2007). Data on reliability (internal consistency and test-retest) and validity (convergent and discriminant) show the ASI-3 to have sound psychometric properties. Normative ASI-3 data reveal that women scored higher than men on the ASI-3 Physical Concerns subscale and on the ASI-3 total score. Implications of these results on psychopathology and clinical psychology are discussed.
\end{abstract}

Keywords: ASI-3, Anxiety sensitivity, anxiety disorders, psychopathology, assessment, confirmatory factor analysis, EQS, PANAS, SA-45, SCL-90.

\section{INTRODUCCIÓN}

La sensibilidad a la ansiedad (SA), o miedo a las sensaciones de ansiedad (Reiss, Peterson, Gursky y McNally, 1986), es uno de los constructos psicológicos de mayor relevancia y

Recibido: 22 diciembre 2006; aceptado 15 febrero 2007.

Correspondencia: Bonifacio Sandín, Universidad Nacional de Educación a Distancia, Facultad de Psicología, Juan del Rosal 10 (Ciudad Universitaria), 28040 Madrid. Correo-e: bsandin@psi.uned.es aplicación en psicopatología y psicología clínica, fundamentalmente por su relación con los trastornos asociados a la ansiedad y el pánico (Reiss, 1991; Sandín, 1999, 2005a; Sandín, Chorot, Santed y Valiente, 2002a; Sandín, Chorot, Valiente y Santed, 2002b; Sandín, Rodero, Santed y García-Campayo, 2006; Taylor, 1999; Valiente, Sandín y Chorot, 2002). Su evaluación se ha llevado a cabo básicamente a través de la escala de 16 items denominada Anxiety Sensitivity Index (ASI; Reiss, et. al., 
1986), cuya versión española ha sido validada por nuestro grupo de investigación (p.ej., Sandín, Chorot, Valiente, Santed, y Lostao, 2004a; Sandín, Valiente, Chorot, y Santed, 2005).

A pesar de la gran aceptación general de esta escala, en los últimos años se han señalado algunas deficiencias a la misma relacionadas con la inconsistencia de su estructura factorial y validez (Taylor, 1999). Básicamente el problema reside en que, siendo la sensibilidad a la ansiedad un constructo de naturaleza multidimensional (i.e., temor a los síntomas de activación física, de ansiedad social, y de descontrol cognitivo), la ASI fue construida como medida unidimensional. Esto es, la principal debilidad de la ASI reside en su validez de contenido. Así, mientras que la dimensión de miedo a las sensaciones físicas está bien representada ( 8 items), la evaluación de las dos restantes dimensiones (social y cognitiva) es más cuestionable, tanto por el número de items (4 items cada dimensión) como por la validez de estos (algunos son ambiguos en lo que concierne a la dimensión a que deben ser asignados). En un intento de solucionar este problema, Taylor y Cox (1998) construyeron la ASI-R, una forma ampliada (36 items) y modificada de la original ASI. Aunque esta escala ha sido validada en distintos países, particularmente a través del estudio transcultural de Zvolensky et al. (2003), se trata de una prueba excesivamente larga que incluye diversas dimensiones, unas replicadas y otras no por los diferentes estudios (Deacon, Abramowitz, Woods y Tolin, 2003; Sandín, Valiente, Chorot y Santed, 2004b; Taylor y Cox, 1998; Zvolensky et al., 2003).

El problema se ha solucionado recientemente construyendo una nueva escala breve de sensibilidad a la ansiedad, la Anxiety Sensitivity Index-3 (ASI-3) a partir de una selección de items de la ASI-R. La ASI-3 evalúa mediante 18 items, y de forma equilibrada (6 items para cada dimensión), las tres dimensiones más consistentemente replicadas en los estudios sobre la sensibilidad a la ansiedad (i.e., las dimensiones física, cognitiva y social). La construcción de esta nueva escala se ha llevado a cabo por un amplio grupo internacional de investigadores liderado por Steven Taylor, en el que ha colabo- rado nuestro equipo de investigación de la UNED (para información sobre la construcción e inicial validación internacional de la ASI-3, véase Taylor et al., 2007). Aunque en esta primera publicación sobre la ASI-3 se presenta amplia información sobre el desarrollo, estructura, fiabilidad y validez de la escala, con evidencia de validación transcultural de la misma (datos con muestras de Canadá, España, Estados Unidos, Francia, Holanda y México), la información concerniente a la versión española se limita básicamente a la convergencia de la estructura factorial y la fiabilidad (consistencia interna), así como también a la contrastación de la superioridad de la ASI-3 respecto a la antigua ASI.

La finalidad del presente estudio consiste en proporcionar información específica sobre la validación de la versión española de la ASI-3 (véase el Anexo I). Esta información incluye evidencia sobre la estructura factorial de la escala, la fiabilidad (consistencia interna y estabilidad temporal), la validez y datos normativos. En varios aspectos, se pretende proporcionar evidencia sobre cuestiones aún no abordadas en el estudio original de Taylor et al. (2007), tales como los relativos al análisis factorial exploratorio de la escala (la selección de los items de la ASI-3 se efectuó racionalmente partiendo de los items de la ASI-R), la fiabilidad y la validez (estabilidad temporal, datos sobre validez convergente y discriminante, etc.), e información sobre datos normativos (p.ej., diferencias entre varones y mujeres). En lo que concierne a su estructura, asumimos una estructura factorial de tres factores correlacionados que deberían representar las dimensiones física, cognitiva y social de la sensibilidad a la ansiedad (primera hipótesis). Basándonos en estudios previos sobre la sensibilidad a la ansiedad (Sandín et al., 2004a; Zinbarg, Barlow y Brown, 1997), aparte de estos tres factores primarios, debería existir un factor general reflejo de la correlación entre los factores primarios y de una estructura jerárquica de la sensibilidad a la ansiedad (segunda hipótesis). La fiabilidad de la ASI-3 (tanto para la escala total como para sus dimensiones) debería ser entre buena y excelente, tanto en relación con su estructura interna como con su estabilidad temporal (ter- 
cera hipótesis). Los datos normativos deberían reflejar puntuaciones en línea con los datos pasados sobre sensibilidad a la ansiedad, p.ej., exhibiendo superiores puntuaciones la mujer que el hombre (cuarta hipótesis). Finalmente, la ASI-3 se relacionará de forma más específica con los constructos más cercanos (o similares) que con los más alejados o diferentes (validez convergente y discriminante) (quinta hipótesis).

\section{MÉTODO}

\section{Participantes}

La muestra total de participantes consistió en 582 estudiantes universitarios pertenecientes a la Universidad Nacional de Educación a Distancia y varias universidades de Madrid. La muestra estaba constituida por 403 mujeres $(69 \%)$ y 179 varones $(31 \%)$. La media de edad del grupo de mujeres era de 24,6 años $(D T=7,4)$ y la del grupo de varones de 27,1 años $(D T=8,9)$, siendo significativas las diferencias entre ambos grupos $[t(580)=3,5, p<0,01]$. Los participantes cumplimentaron en una ocasión de forma colectiva (en grupos de 20-40 alumnos aproximadamente) en diversos centros docentes universitarios los cuestionarios que se indican a continuación. Ochenta y cinco participantes contestaron a la ASI-3 en una segunda ocasión un mes más tarde.

\section{Pruebas psicológicas}

Índice de Sensibilidad a la Ansiedad-3 [Anxiety Sensitivity Index-3, ASI-3] (Taylor et al., 2007). Se aplicó la versión española (véase el Anexo I). Consta de 18 items que el participante debe contestar indicando el grado en que suele experimentar cada uno de los enunciados según una escala tipo Likert, pudiendo variar entre «Nada o casi nada» (0) y «Muchísimo» (4). Los items hacen referencia a reacciones de miedo/ansiedad ante la experiencia de síntomas de tipo físico (p.ej., «Cuando siento opresión en el pecho, me asusta no poder respirar bien»), síntomas de descontrol cognitivo (p.ej., «Cuando me resulta difícil pensar con claridad, me preocupa que me esté ocurriendo algo grave»), y síntomas observables socialmente («Tengo miedo a sonrojarme delante de la gente»). La ASI-3 incluye tres subescalas de sensibilidad a la ansiedad (física, cognitiva y social), cada una de las cuales está constituida por 6 items. Los primeros datos sobre construcción, estructura, fiabilidad y validez de la ASI-3 fueron publicados por Taylor et al. (2007).

Escalas de Afecto Positivo y Negativo [Positive and Negative Affect Schedule, PANAS] (Watson, Clark y Tellegen, 1988). Se aplicó la versión española validada por Sandín et al. (1999). Consta de 20 items, 10 referidos al afecto positivo y 10 al afecto negativo. Cada ítem se puntúa según una escala Likert de 1 («Nada o casi nada») a 5 («Muchísimo»).

Cuestionario de Síntomas Psicopatológicos [Symptom Assessment-45 Questionnaire, SA45] (Davison et al., 1997). El SA-45 es una forma abreviada del SCL-90. El sujeto debe indicar hasta qué punto ha experimentado cada uno de los 45 síntomas durante la última semana, según una escala Likert entre 0 ( «Nada en absoluto) y 4 («Mucho o extremadamente»). El cuestionario evalúa las mismas dimensiones que el SCL-90: somatización, obsesión-compulsión, sensibilidad interpersonal, depresión, ansiedad, hostilidad, ansiedad fóbica (agorafobia), ideación paranoide, y psicoticismo. Empleamos la versión española validada recientemente por nuestro grupo (Sandín, Valiente, Chorot y Santed, 2007).

\section{RESULTADOS}

\section{Análisis factorial exploratorio de la ASI-3}

Antes de efectuarse el análisis factorial se comprobó si los datos eran estadísticamente adecuados para ello. Tanto la prueba de la medida de adecuación muestral de Kaiser-MeyerOlkin $(=0,91)$, como prueba de esfericidad de Bartlet $\left[\chi^{2}(153)=5023,9, p<0,001\right]$, sugerían dicha adecuación. En la Tabla 1 presentamos los datos relativos al análisis factorial exploratorio (análisis de componentes principales y 
rotación oblimin) (aplicamos también análisis factorial de ejes principales y los resultados fueron similares). Aunque el número de factores (3) era el recomendado por el modelo teórico, tanto la visualización gráfica de los valores propios (test de scree) como el criterio de Kaiser (sólo había tres factores con valores propios por encima de la unidad) sugerían claramente una estructura de tres factores. El porcentaje de varianza total explicada era notable (cerca del 60\%).

Puede observarse que se trata de una estructura factorial claramente delimitada con una configuración muy robusta. Los tres factores obtenidos (cognitivo, social y físico) aparecen muy bien definidos, estando todas las saturaciones (relevantes al factor) por encima de 0,45 (la mayoría superan el valor de 0,70). Así mismo, no existen items hiperplanos ni transaturaciones (saturaciones elevadas en más de un factor). El primer factor (cognitivo) está configurado por los items 2, 5, 10, 14, 16 y 18 y representa la dimensión cognitiva de sensibilidad a la ansiedad. El segundo factor (social) viene definido por los items 1, 6, 9, 11, $13 \mathrm{y}$ 17; representa la dimensión social de la sensibilidad a la ansiedad. Finalmente, el tercer factor configura la dimensión física de sensibilidad a la ansiedad (items 3, 4, 7, 8, 12 y 15). Estos datos son totalmente consistentes con la definición de las dimensiones de la ASI-3 y con los datos previos presentados por Taylor et al. (2007).

\section{Análisis factorial de la estructura de orden superior de la ASI-3}

Como cabría esperar, las correlaciones entre los tres componentes resultaron ser moderadas, oscilando entre 0,35 y 0,51 . Estas correlaciones sugieren la existencia de un posible factor de orden superior. El análisis de la estructura de orden superior de la ASI-3 se llevó a cabo factorizando las puntuaciones factoriales de los tres factores primarios rotados oblicuamente. Los resultados del análisis de componentes principales (similares a los obtenidos mediante el análisis de ejes principales) sugerían la existencia de un factor de orden superior, apoyan- do por tanto una estructura jerárquica de la ASI-3. Dicho factor explicaba el $61 \%$ de la varianza total (las saturaciones de los factores primarios en este factor general variaron entre $0,51$ y 0,67$)$.

\section{Análisis factorial confirmatorio de la ASI-3}

Mediante análisis factorial confirmatorio (ecuaciones estructurales) queríamos probar que el modelo de tres factores era superior a otras alternativas sugeridas previamente para la ASI y/o ASI-R, tales como el modelo de dos factores (Sandín et al., 2002a; Zvolensky et al., 2003) o el modelo de un factor (Sandín, Chorot y McNally, 1996). Además, era necesario contrastar la hipótesis de que la estructura jerárquica (tres factores primarios y un factor de orden superior) se ajustaba mejor a los datos que una estructura de sólo tres factores (Sandín et al., 2004a; Zinbarg et al., 1997). Para ello sometimos a prueba los cuatro modelos estructurales que indicamos en la Tabla 2: (1) modelo de tres factores correlacionados, (2) modelo de tres factores primarios y un factor de orden superior (modelo jerárquico), (3) modelo de dos factores correlacionados, y (4) modelo de un solo factor.

Los análisis se efectuaron con el programa de ecuaciones estructurales EQS (Bentler, 2005). La definición de los modelos se realizó del siguiente modo. El modelo de tres factores se construyó de acuerdo con la asignación de los items a cada factor indicada previamente por Taylor et al. (2007), la cual coincide exactamente con nuestros resultados del análisis factorial exploratorio (véase la Tabla 1); cada una de las 18 variables observadas debía saturar sólo en uno de los tres factores, permitiéndose la correlación entre éstos. El segundo modelo supone una relación similar entre las 18 variables observadas y las tres variables latentes, si bien estas últimas deben saturar, así mismo, en una cuarta variable latente (factor de orden superior), lo que le convierte en un modelo de estructura jerárquica (los datos exploratorios indicados atrás sugieren esta estructura). El tercer modelo se definió partiendo de la hipótesis sugerida por Sandín et al. 
Tabla 1. Análisis factorial exploratorio (matriz de configuración) de la ASI-3 (análisis de componentes principales, rotación oblimin; $N=582$ )

\begin{tabular}{|c|c|c|c|c|}
\hline Ítem ASI-3 & $\begin{array}{l}\text { Factor 1: } \\
\text { Cognitivo }\end{array}$ & $\begin{array}{c}\text { Factor 2: } \\
\text { Social }\end{array}$ & $\begin{array}{l}\text { Factor 3: } \\
\text { Físico }\end{array}$ & $h^{2}$ \\
\hline 1. Para mí es importante no dar la impresión de estar nervioso/a & 0,14 & 0,78 & 0,01 & 0,55 \\
\hline $\begin{array}{l}\text { 2. Cuando no puedo mantener mi mente concentrada en una tarea, } \\
\text { siento la preocupación de que podría estar volviéndome loco/a }\end{array}$ & 0,93 & 0,01 & 0,09 & 0,78 \\
\hline Me asusto cuando mi corazón late de forma rápida & 0,07 & 0,01 & 0,77 & 0,55 \\
\hline $\begin{array}{l}\text { 4. Cuando siento malestar en el estómago, me preocupa estar } \\
\text { seriamente enfermo/a }\end{array}$ & 0,10 & 0,08 & $\mathbf{0 , 5 1}$ & 0,35 \\
\hline $\begin{array}{l}\text { 5. Me asusto cuando soy incapaz de mantener mi mente concentrada } \\
\text { en una tarea }\end{array}$ & 0,76 & 0,04 & 0,03 & 0,64 \\
\hline $\begin{array}{l}\text { 6. Cuando tiemblo en presencia de otras personas, me da miedo } \\
\text { lo que puedan pensar de mí }\end{array}$ & 0,26 & 0,72 & 0,06 & 0,68 \\
\hline Cuando siento opresión en el pecho, me asusta no poder respirar bien & 0,06 & 0,12 & 0,72 & 0,66 \\
\hline $\begin{array}{l}\text { 8. Cuando siento dolor en el pecho, me preocupa que vaya a darme } \\
\text { un ataque cardíaco }\end{array}$ & 0,13 & 0,13 & $\mathbf{0 , 8 3}$ & 0,74 \\
\hline 9. Me preocupa que otras personas noten mi ansiedad & 0,18 & 0,71 & 0,02 & 0,65 \\
\hline $\begin{array}{l}\text { 10. Cuando tengo la sensación de que las cosas no son reales, } \\
\text { me preocupa que pueda estar mentalmente enfermo/a }\end{array}$ & $\mathbf{0 , 5 1}$ & 0,08 & 0,17 & 0,43 \\
\hline 11. Tengo miedo a sonrojarme delante de la gente & 0,07 & 0,76 & 0,01 & 0,53 \\
\hline $\begin{array}{l}\text { 12. Cuando noto que mi corazón da un salto o late de forma irregular, } \\
\text { me preocupa que algo grave me esté ocurriendo }\end{array}$ & 0,11 & 0,02 & 0,79 & 0,75 \\
\hline $\begin{array}{l}\text { 13. Cuando comienzo a sudar en una situación social, me da miedo } \\
\text { que la gente piense negativamente de mí }\end{array}$ & 0,09 & 0,74 & 0,03 & 0,63 \\
\hline $\begin{array}{l}\text { 14. Cuando mis pensamientos parecen acelerarse, me preocupa que pueda } \\
\text { volverme loco/a }\end{array}$ & 0,71 & 0,03 & 0,04 & 0,56 \\
\hline $\begin{array}{l}\text { 15. Cuando siento opresión en la garganta, me preocupa que pueda } \\
\text { atragantarme y morir }\end{array}$ & 0,22 & 0,11 & 0,67 & 0,59 \\
\hline $\begin{array}{l}\text { 16. Cuando me resulta difícil pensar con claridad, me preocupa que } \\
\text { me esté ocurriendo algo grave }\end{array}$ & $\mathbf{0 , 8 1}$ & 0,05 & 0,01 & 0,70 \\
\hline 17. Pienso que me resultaría horrible si me desmayase en público & 0,09 & 0,46 & 0,14 & 0,34 \\
\hline $\begin{array}{l}\text { 18. Cuando mi mente se queda en blanco, me preocupa que me esté } \\
\text { ocurriendo algo terriblemente malo }\end{array}$ & 0,72 & 0,01 & 0,11 & 0,62 \\
\hline Autovalores (iniciales) & 7,14 & 2,06 & 1,44 & \\
\hline$\%$ Varianza explicada $($ total $=59,14 \%)$ & 39,71 & 11,43 & 8,00 & \\
\hline
\end{tabular}

Nota. En negrita se indican los pesos factoriales $\geq 0,40 . h^{2}=$ comunalidad.

Tabla 2. Índices de bondad de ajuste para los diferentes modelos relacionados con la estructura factorial de la ASI-3, basados en los análisis factoriales confirmatorios EQS $(N=582)$

\begin{tabular}{|c|c|c|c|c|c|c|c|}
\hline Modelo de análisis factorial confirmatorio de la ASI-3 & $S-B \chi^{2}(g l)$ & $\chi^{2} / g l$ & NNFI & CFI & SRMR & RMSEA & AIC \\
\hline Modelo 1: Tres factores correlacionados & $343,18(132)$ & 2,60 & 0,92 & 0,93 & 0,05 & 0,05 & 79,18 \\
\hline Modelo 2: Tres factores y un factor de orden superior & $323,01(129)$ & 2,50 & 0,93 & 0,94 & 0,05 & 0,05 & 65,00 \\
\hline Modelo 3: Dos factores correlacionados & $489,64(134)$ & 3,65 & 0,74 & 0,77 & 0,08 & 0,09 & 489,64 \\
\hline Modelo 4: Un factor & $1.060,26(135)$ & 7,85 & 0,62 & 0,67 & 0,10 & 0,11 & 790,26 \\
\hline
\end{tabular}

Nota. Índices corregidos (método robusto). $S$ - $B \chi^{2}=\chi^{2}$ escalado de Satorra-Bentler. NNFI = non-normed fit index; $\mathrm{CFI}=$ comparative fit index; SRMR = standardized root mean square residual; RMSEA = root mean square error of approximation; $\mathrm{AIC}=$ Akaike's information criterion. 
(2002a) y Zvolensky et al. (2003), según la cual los elementos de tipo social y cognitivo se asocian a un único factor. En consecuencia, el modelo se construyó con dos factores, en uno de ellos saturaban las variables de la ASI-3 de naturaleza física y en un segundo factor el resto de variables (variables de naturaleza cognitiva o social). Finalmente, construimos un cuarto modelo en el que todas las variables de la ASI saturaban en un único factor de sensibilidad a la ansiedad.

Todos los modelos eran de tipo congenérico. Las varianzas de los términos de error fueron especificadas como parámetros libres. No se permitió la covariación entre los residuos. Puesto que los datos basados en cuestionarios tipo Likert pueden presentar problemas de distribución normal, se utilizó el método de estimación ML-robusto, el cual proporciona estadísticos robustos a partir de la prueba escalada de $\chi^{2}$ de Satorra-Bentler $\left(S-B \chi^{2}\right)$ y errores estándar robustos, corrigiendo los errores debidos a una posible violación del principio de normalidad. La aplicación de este método significa que los valores calculados son válidos incluso aunque se violase la asunción de normalidad en el método de estimación.

Empleamos los siguientes indicadores de bondad de ajuste: (1) $S-B \chi^{2}\left(\chi^{2}\right.$ de Satorra-Bentler), (2) $S$ - $B \chi^{2}$ dividido por los grados de libertad (cocientes $\leq 5,0$ indican buen ajuste; a menor índice mejor ajuste), (3) NNFI (non-normed fit index), (4) CFI (comparative fit index), (5) SRMR (standardized root mean-square residual), (6) RMSEA (root mean square error of approximation), y (7) AIC (Akaike's information criterion). Se considera un ajuste aceptable si los valores de NNFI y CFI se acercan a 0,90, SRMR $\leq 0,08, y$ RMSEA $\leq 0,06$. El AIC es un indicador relativo; en ausencia de otros criterios definitorios, el mejor modelo es el que posea un AIC más bajo.

Tal y como puede observarse en la Tabla 2, los dos primeros modelos son claramente superiores a los modelos 3 y 4 . Más aun, mientras que los modelos 1 y 2 se ajustan bastante bien a los datos, los dos últimos modelos, especialmente el modelo 4, presentan índices en general pobres. De aquí se deriva que la ASI-3 está bien definida como una escala de evaluación tridimensional de la sensibilidad a la ansiedad. Una cuestión importante, no obstante, consistiría en determinar cual de los dos modelos basados en una estructura tridimensional (i.e., modelos 1 y 2) exhibe mejor ajuste. Los índices absolutos de ajuste que presentamos en la Tabla 2 sugieren cierta superioridad del modelo jerárquico (sólo son iguales para los índices SRMR y RMSEA). Así mismo, los valores del AIC sugieren mejor ajuste en este último modelo. Puesto que los modelos son anidados, calculamos el cambio entre ambos modelos en el $S$ - $B \chi^{2}$, siendo $\Delta S-B \chi^{2}(3)=20,17, \mathrm{p}<0,01$, lo que sugiere, una vez más, una superioridad significativa del modelo jerárquico sobre el modelo de tres factores primarios.

\section{Fiabilidad}

En lo que concierne a la consistencia interna de la escala podemos decir que su fiabilidad es excelente, tal y como se deriva de los elevados coeficientes alfa, tanto el de la escala total como los referidos a las tres subescalas (véase la Tabla 3). Así mismo, las correlaciones corregidas ítemsubescala tienden a ser entre moderadas-altas y elevadas, lo que sugiere que todos y cada uno de los items de la ASI-3 representan adecuadamente el constructo de sensibilidad a la ansiedad y sus respectivas dimensiones. Las correlaciones entre las tres subescalas varían entre 0,42 y 0,59 , correlacionando todas ellas de forma similar con la ASI-3 total (véase la Tabla 4).

La estabilidad temporal (un mes) de la ASI3 también ha resultado ser excelente, tal y como se desprende de las elevadas correlaciones testretest obtenidas, tanto para la escala total como para las tres subescalas (véase la Tabla 4).

\section{Datos normativos}

Las medias y desviaciones típicas se indican de forma separada para los grupos de varones y mujeres, y para la muestra total, así como también para cada uno de los elementos de la escala, para cada subescala, y para la escala total (véase la Tabla 3). Puesto que existían diferencias significativas en edad entre varones 
y mujeres, se controló el efecto de esta variable en todos los análisis realizados para comparar las puntuaciones entre ambos grupos.

Para examinar las posibles diferencias entre los grupos de varones y mujeres en las puntuaciones a los 18 items de la ASI-3 aplicamos un diseño de MANOVA de un factor (sexo) y 18 variables dependientes (i.e., los 18 items de la escala). Los resultados indicaron la existencia de efectos significativos globales del factor sexo [Hotelling $F(18,562)=3,8, p<0,001]$. Posteriormente se calcularon los efectos univariados de la variable sexo para cada una de las variables dependientes (en la Tabla 3 indicamos cada prueba de $F$ y su significación estadística). Como podemos observar, las mujeres obtienen puntuaciones más elevadas que los varones únicamente en los items 3 («Me asusto cuando mi corazón late de forma rápida»), 7 («Cuado siento opresión en el pecho, me asusta no poder respirar bien»), y 9 («Me preocupa que otras personas noten mi ansiedad»).

Aplicamos un diseño de MANOVA similar, si bien de 3 variables dependientes (las tres subescalas de la ASI-3). También observamos efectos significativos globales, siendo significativa la traza de Hotelling $[F(3,577)=3,4, p<0,05]$. Tras calcular los efectos univariados, sólo se constataron diferencias significativas entre varones y mujeres en la subescala física de sensibilidad a la ansiedad (las mujeres puntúan de forma más elevada que los hombres) (véase la Tabla 3).

Con objeto de analizar las posibles diferencias sexuales en las puntuaciones a la escala

Tabla 3. Análisis de items y subescalas de la ASI-3 (correlación ítem-subescala corregida, coeficientes $\alpha$, medias y DTs).

\begin{tabular}{lccccc}
\hline & $\begin{array}{c}\text { Total } \\
(N=582)\end{array}$ & $\begin{array}{c}\text { Varones } \\
(N=179)\end{array}$ & $\begin{array}{c}\text { Mujeres } \\
(N=403)\end{array}$ & $\begin{array}{c}\text { Varones vs. } \\
\text { Mujeres }\end{array}$ & $\begin{array}{c}\text { Total } \\
(N=582)\end{array}$ \\
\hline Ítem de la ASI-3 (rango 0-4) & Media $(D T)$ & Media $(D T)$ & Media $(D T)$ & $F(1,579)$ & Ítem-subescala \\
\hline 1 & $1,6(1,1)$ & $1,7(1,1)$ & $1,6(1,1)$ & 1,9 & 0,54 \\
2 & $0,3(0,7)$ & $0,3(0,7)$ & $0,3(0,7)$ & $<1$ & 0,78 \\
3 & $1,0(0,8)$ & $0,8(0,8)$ & $1,1(0,8)$ & $12,4 * * *$ & 0,57 \\
4 & $0,6(1,5)$ & $0,4(0,7)$ & $0,7(1,7)$ & 2,6 & 0,35 \\
5 & $0,5(0,8)$ & $0,5(0,7)$ & $0,6(0,8)$ & $<1$ & 0,68 \\
6 & $0,8(0,9)$ & $0,8(0,9)$ & $0,9(0,9)$ & $<1$ & 0,71 \\
7 & $1,1(1,0)$ & $0,9(0,9)$ & $1,3(1,0)$ & $21,9 * * *$ & 0,67 \\
8 & $0,6(0,9)$ & $0,6(0,9)$ & $0,6(0,9)$ & $<1$ & 0,71 \\
9 & $1,1(1,0)$ & $0,9(0,9)$ & $1,2(1,0)$ & $8,9 *$ & 0,69 \\
10 & $0,6(0,9)$ & $0,6(0,8)$ & $0,6(0,9)$ & 1,2 & 0,53 \\
11 & $1,0(1,0)$ & $0,9(0,9)$ & $1,1(1,1)$ & 2,7 & 0,55 \\
12 & $0,8(0,9)$ & $0,7(0,9)$ & $0,8(0,9)$ & 2,4 & 0,74 \\
13 & $1,2(1,0)$ & $1,0(1,0)$ & $1,2(1,1)$ & 3,5 & 0,67 \\
15 & $0,5(0,9)$ & $0,4(0,8)$ & $0,5(0,9)$ & 1,3 & 0,64 \\
16 & $0,4(0,8)$ & $0,5(0,8)$ & $0,4(0,8)$ & 1,1 & 0,61 \\
18 & $0,5(0,8)$ & $0,5(0,8)$ & $0,6(0,8)$ & $<1$ & 0,75 \\
& $1,1(1,1)$ & $1,0(1,1)$ & $1,2(1,1)$ & 2,5 & 0,45 \\
& $0,3(0,7)$ & $0,3(0,6)$ & $0,3(0,7)$ & 2,9 & 0,67
\end{tabular}

Subescalas de la ASI-3

\begin{tabular}{lrrrrr} 
(rango 0-24) & Media $(D T)$ & Media $(D T)$ & Media $(D T)$ & $F(1,579)$ & Coeficiente $\alpha$ \\
\hline ASI-3 Física & $4,5(4,1)$ & $3,7(3,5)$ & $4,8(4,4)$ & $9,5^{* *}$ & 0,84 \\
ASI-3 Cognitiva & $2,7(3,5)$ & $2,4(3,3)$ & $2,8(3,7)$ & 1,4 & 0,87 \\
ASI-3 Social & $6,9(4,6)$ & $6,3(4,5)$ & $7,1(4,7)$ & 2,7 & 0,83 \\
ASI-3 Total & $14,1(9,6)$ & $12,8(9,3)$ & $14,7(9,9)$ & $7,4 * *$ & 0,91 \\
\hline
\end{tabular}

Nota. Para las comparaciones múltiples entre varones y mujeres se aplicó la corrección de Bonferroni. Las correlaciones ítemsubescala corresponden a las correlaciones entre cada ítem y su correspondiente subescala de la ASI-3 si se elimina el elemento. $* p<0,05 ; * * p<0,01 ; * * * p<0,001$. 
Tabla 4. Correlaciones entre las subescalas de la ASI-3 y consistencia temporal (test-retest) $(N=582)^{\mathrm{a}}$

\begin{tabular}{lcccc}
\hline Subescalas ASI-3 & ASI-3 Física & ASI-3 Cognitiva & ASI-3 Social & ASI-3 Total \\
\hline ASI-3 Física & $(0,85)$ & & & \\
ASI-3 Cognitiva & 0,59 & $(0,83)$ & $(0,84)$ & \\
ASI-3 Social & 0,42 & 0,49 & 0,80 & $(0,85)$ \\
ASI-3 Total & 0,82 & 0,83 & & \\
\hline
\end{tabular}

Nota. ${ }^{\text {a }}$ Para las correlaciones test-retest $N=85$. Entre paréntesis se indican las correlaciones test-retest.

Tabla 5. Percentiles (puntos de corte) para las puntuaciones en las subescalas de la ASI-3 y en la escala total

\begin{tabular}{|c|c|c|c|c|c|c|c|c|c|c|c|c|}
\hline \multirow[b]{2}{*}{ Percentil } & \multicolumn{3}{|c|}{ ASI-3 Física } & \multicolumn{3}{|c|}{ ASI-3 Cognitiva } & \multicolumn{3}{|c|}{ ASI-3 Social } & \multicolumn{3}{|c|}{ ASI-3 Total } \\
\hline & $\sigma^{x}$ & 우 & $\sigma^{x}+q$ & $\sigma^{7}$ & 우 & $\sigma^{x}+q$ & $0^{7}$ & 우 & $\sigma^{x}+q$ & $0^{7}$ & ㅇ & $0^{x}+$ Q \\
\hline 5 & 0 & 0 & 0 & 0 & 0 & 0 & 0 & 1 & 1 & 0 & 3 & 3 \\
\hline 10 & 0 & 1 & 0 & 0 & 0 & 0 & 0 & 1 & 1 & 1 & 4 & 4 \\
\hline 15 & 0 & 1 & 1 & 0 & 0 & 0 & 1 & 2 & 2 & 3 & 5 & 5 \\
\hline 20 & 0 & 1 & 1 & 0 & 0 & 0 & 1 & 3 & 3 & 4 & 6 & 6 \\
\hline 25 & 1 & 2 & 2 & 0 & 0 & 0 & 2 & 3 & 3 & 4 & 7 & 7 \\
\hline 30 & 1 & 2 & 2 & 0 & 0 & 0 & 3 & 4 & 4 & 5 & 8 & 8 \\
\hline 35 & 1 & 2 & 2 & 0 & 1 & 1 & 3 & 5 & 5 & 7 & 9 & 9 \\
\hline 40 & 2 & 3 & 3 & 0 & 1 & 1 & 4 & 5 & 5 & 8 & 10 & 10 \\
\hline 45 & 3 & 3 & 3 & 1 & 1 & 1 & 5 & 6 & 6 & 9 & 11 & 11 \\
\hline 50 & 3 & 4 & 4 & 1 & 1 & 1 & 5 & 6 & 6 & 9 & 13 & 11 \\
\hline 55 & 4 & 4 & 4 & 1 & 2 & 2 & 6 & 7 & 7 & 10 & 14 & 13 \\
\hline 60 & 4 & 5 & 4 & 2 & 2 & 2 & 6 & 7 & 7 & 11 & 15 & 14 \\
\hline 65 & 5 & 5 & 5 & 2 & 3 & 2 & 7 & 8 & 8 & 13 & 16 & 15 \\
\hline 70 & 5 & 6 & 5 & 3 & 3 & 3 & 8 & 9 & 9 & 14 & 18 & 17 \\
\hline 75 & 6 & 7 & 6 & 3 & 4 & 4 & 8 & 10 & 10 & 18 & 20 & 19 \\
\hline 80 & 6 & 7 & 7 & 5 & 5 & 5 & 10 & 11 & 11 & 20 & 22 & 22 \\
\hline 85 & 7 & 8 & 8 & 6 & 6 & 6 & 11 & 12 & 12 & 23 & 25 & 25 \\
\hline 90 & 9 & 10 & 10 & 8 & 8 & 7 & 13 & 13 & 14 & 27 & 29 & 28 \\
\hline 95 & 13 & 13 & 12 & 10 & 11 & 10 & 15 & 16 & 15 & 35 & 32 & 32 \\
\hline
\end{tabular}

Nota. Varones $\left(\sigma^{7}\right) N=209 ;$ mujeres (O) $\left.N=373\right)$; muestra total $\left(\sigma^{7}+\right.$ Q $) N=582$.

total, aplicamos un diseño de ANOVA de un factor (sexo), siendo la variable dependiente la ASI-3 total. Como se indica en la Tabla 3, las mujeres obtuvieron puntuaciones significativamente más elevadas que los varones.

Finalmente, con fines descriptivos calculamos los datos normativos en percentiles para la escala total y las tres subescalas, separando los valores correspondientes a los grupos de varones y mujeres, y a la muestra total (véase la Tabla 5).

\section{Validez convergente y discriminante}

Con objeto de proporcionar información preliminar sobre la validez convergente y discrimi- nante de la versión española de la ASI-3, en la Tabla 6 se indican las correlaciones entre esta escala y otras variables de interés clínico y psicopatológico (afectividad positiva y negativa, y síntomas de psicopatología). En lo que concierne a la correlación entre la ASI-3 y el afecto, las correlaciones son bajas y negativas o insignificantes con el afecto positivo y moderadas y positivas con el afecto negativo. Este patrón es consistente con el constructo de sensibilidad a la ansiedad, que presupone cierta relación positiva con el rasgo de ansiedad y el afecto negativo, y una relación nula con el afecto positivo (Taylor, 1999).

En la Tabla 6 presentamos las correlaciones de Pearson entre las variables de sensibilidad a 
Tabla 6. Correlaciones entre la ASI-3 (total y subescalas) y las variables de afectividad (PANAS) y psicopatología $(\mathrm{SA}-45)(N=582)$

\begin{tabular}{|c|c|c|c|c|}
\hline Variables de afecto y psicopatología & ASI-3 Física & ASI-3 Cognitiva & ASI-3 Social & ASI-3 Total \\
\hline \multicolumn{5}{|l|}{ PANAS } \\
\hline Afecto positivo & 0,01 & $-0,05$ & 0,07 & 0,02 \\
\hline Afecto negativo & 0,28 & 0,28 & 0,49 & 0,43 \\
\hline \multicolumn{5}{|l|}{ SA-45 } \\
\hline Depresión & $0,28(0,09)$ & $0,41(0,21)$ & $0,40(0,16)$ & $0,46(0,20)$ \\
\hline Hostilidad & $0,17(0,01)$ & $0,29(0,14)$ & $0,19(0,03)$ & $0,27(0,03)$ \\
\hline Sensibilidad Interpersonal & $0,30(0,15)$ & $0,36(0,20)$ & $0,47(0,30)$ & $0,49(0,30)$ \\
\hline Somatización & $0,27(0,12)$ & $0,36(0,21)$ & $0,35(0,15)$ & $0,42(0,22)$ \\
\hline Ansiedad & $0,33(0,13)$ & $0,47(0,24)$ & $0,39(0,12)$ & $0,51(0,22)$ \\
\hline Psicoticismo & $0,20(0,07)$ & $0,33(0,21)$ & $0,31(0,11)$ & $0,35(0,19)$ \\
\hline Obsesión-Compulsión & $0,32(0,15)$ & $0,38(0,23)$ & $0,41(0,25)$ & $0,47(0,28)$ \\
\hline Ansiedad Fóbica (Agorafobia) & $0,29(0,17)$ & $0,40(0,24)$ & $0,41(0,25)$ & $0,47(0,32)$ \\
\hline Ideación Paranoide & $0,25(0,12)$ & $0,31(0,23)$ & $0,37(0,14)$ & $0,33(0,18)$ \\
\hline SA-45-total & $0,35(0,17)$ & $0,48(0,32)$ & $0,48(0,27)$ & $0,56(0,34)$ \\
\hline
\end{tabular}

Nota . PANAS = Positive and Negative Affect Scales; SA-45 = Symptom Assessment-45 Questionnaire. Entre paréntesis se indican las correlaciones parciales controlando el efecto del afecto negativo. Correlaciones a partir de $0,12, p<0,01$.

la ansiedad y las variables psicopatológicas (SA-45). Dadas las significativas correlaciones que existían entre la sensibilidad a la ansiedad y el afecto negativo, presentamos también las correlaciones parciales entre dichas variables controlando el efecto de la afectividad negativa. Podemos observar un importante decremento en el valor de las correlaciones al parcializar el afecto negativo.

\section{DISCUSIÓN}

El primer estudio sobre la nueva escala de evaluación de la sensibilidad a la ansiedad (la ASI-3) fue llevado a cabo por Taylor et al. (2007). En dicha investigación se proporciona información sobre la construcción y validación preliminar de la escala, incluyendo datos sobre validación transcultural de la misma relativos a poblaciones de Canadá, España, Estados Unidos, Francia, Holanda y México. Aunque en este estudio se aportan datos preliminares relativos a la validación de la versión española de la ASI-3, se precisan estudios más específicos centrados en el estudio de las propiedades psicométricas de este nuevo cuestionario. En el presente trabajo se han investigado algunas de estas propiedades. Más específicamente, nos hemos centrado en cuatro aspectos fundamentales para la validación de la versión española de la ASI-3: (1) su estructura factorial, (2) su fiabilidad, (3) sus puntuaciones normativas, y (4) su validez

Nuestro análisis factorial exploratorio sugiere de forma clara y concluyente que la ASI-3 es multidimensional, estando constituida por tres factores primarios bien definidos y delimitados, los cuales hacen referencia a las tres dimensiones (o subescalas) propuestas inicialmente por Taylor et al. (2007), esto es, las dimensiones de sensibilidad a la ansiedad física, cognitiva y social. Este es el primer estudio en el que se presentan datos basados en análisis factorial exploratorio, pues en el estudio original de Taylor et al. (2007) utilizamos una aproximación racional para la selección de los items y, posteriormente, una aproximación de análisis estructural (análisis factorial confirmatorio) para la selección de los items definitivos. Además, nuestro análisis factorial exploratorio sugiere la existencia de un factor general que explicaría las correlaciones observadas entre los tres factores primarios.

Partiendo de estos datos, así como también de la evidencia presentada en estudios previos sobre la sensibilidad a la ansiedad (Cintrón, Carter, Suchday, Sbrocco y Gray, 2005; Sandín 
et al., 1996, 2002a, 2004a; Taylor, 1999; Taylor et al., 2007; Zinbarg et al., 1997; Zvolensky et al., 2003), sometimos a prueba varios modelos alternativos de análisis factorial confirmatorio que incluían la consideración de una estructura de la ASI-3 de uno, dos o tres factores, así como también la posibilidad de una estructura jerárquica. En relación con esta cuestión nuestros datos apoyan los aportados en el estudio transcultural de Taylor et al. (2007), en el sentido de que la ASI-3 está constituida por tres dimensiones bien definidas que corresponden a las tres facetas de sensibilidad a la ansiedad indicadas arriba (i.e., física, cognitiva y social). No obstante, los resultados del presente estudio indican, además, que el modelo basado en una estructura jerárquica se ajusta mejor a los datos que la estructura no jerárquica. Es decir, los tres factores primarios de sensibilidad a la ansiedad indicados saturan en un factor general que explicaría un porcentaje de la varianza (común a estos tres factores). Esto indica que la ASI-3 posee un componente común y general de sensibilidad a la ansiedad (común a las tres dimensiones) y tres componentes específicos (i.e., específicos de las dimensiones física, cognitiva y social, respectivamente). Estos resultados son los primeros publicados hasta el momento sobre la estructura jerárquica de la ASI-3, y son consistentes con la evidencia obtenida previamente sobre la estructura jerárquica de la ASI y la ASI-R (Sandín et al., 2004a, 2004b; Stewart, Taylor y Baker, 1997; Taylor, 1999; Taylor y Cox, 1998; Zinbarg et al., 1997). Aunque a veces se ha señalado que podría tratarse de emocionalidad negativa, sin duda una cuestión importante a dilucidar en futuras investigaciones sería la determinación de la naturaleza de este factor general de sensibilidad a la ansiedad.

La existencia de un modelo jerárquico de la sensibilidad a la ansiedad posee importantes implicaciones para la psicopatología y el tratamiento de la ansiedad (Sandín et al., 2004a), y posiblemente también de otros trastornos psicológicos. Por ejemplo, el hecho de que existan tres componentes diferenciados de sensibilidad a la ansiedad, permite derivar subescalas separadas y relacionar estas dimensiones con otros constructos psicólogos de la ansiedad y otras características psicopatológicas. Mientras que ciertos componentes de la sensibilidad a la ansiedad, o bien el factor general, pueden estar etiológicamente más implicados en unos trastornos de ansiedad que en otros, también pueden ser de gran utilidad para estudios sobre la predicción y evolución clínica de los trastornos de ansiedad (p.ej., la mejoría clínica se ha asociado a un marcado decremento de la sensibilidad a la ansiedad). Así por ejemplo, mientras que la dimensión somática parece estar más relacionada con el trastorno de pánico (Zinbarg et al., 1997; Brown, Smits, Powers y Telch, 2003), la dimensión social presenta una relación más estrecha con la fobia social (Zinbarg et al., 1997). Así mismo, se ha sugerido que la faceta cognitiva presenta una cierta asociación con la depresión mayor (Taylor, Koch, Woody y McLean, 1996). Por otra parte, el hecho de que exista un factor general de sensibilidad a la ansiedad, justifica la pertinencia, y posible conveniencia, de emplear la puntuación total de la escala además de las puntuaciones separadas de las tres dimensiones. Parece indudable, por tanto, que la consideración multidimensional y jerárquica de la ASI-3 potenciaría su utilidad como herramienta para la investigación, el diagnóstico y el tratamiento de los trastornos asociados a la ansiedad, y posiblemente de otros trastornos (Cabrera, Urrutia, Vera, Alvarado y Vera-Villaroel, 2005; Gimeno y Ezquerro, 2006; Nuevo, 2005; Sandín, 2005b).

Los datos sobre fiabilidad (consistencia interna) de la ASI-3 están en línea con los presentados en el estudio original (Taylor et al., 2007), siendo los coeficientes alfa excelentes, tanto para la escala total como para las tres subescalas. Además, en el presente estudio presentamos evidencia de la fiabilidad basada en análisis de items, en la que se demuestra que las correlaciones entre los elementos y la subescala correspondiente son apropiadas, lo cual garantiza el empleo separado de estas tres subescalas de sensibilidad a la ansiedad (aparte del posible uso de la escala como puntuación total). Estos datos, por otra parte, contrastan con las deficiencias observadas en torno a la consistencia interna de algunas dimensiones de la ASI (Sandín et al., 2004a, 2005). Los datos presentados en este estudio basados en las correlaciones test-rest sugieren, así mismo, que 
la estabilidad temporal de la ASI-3 es excelente, tanto para la escala total como para sus tres dimensiones. Hasta estos momentos no se habían publicado datos sobre la fiabilidad temporal de la ASI-3, ni tampoco sobre la fiabilidad basada en análisis de items (correlaciones ítemsubescala).

Los resultados sobre puntuaciones normativas (medias y desviaciones típicas) de la ASI3 se hallan en el rango referido por Taylor et al. (2007), tanto para las puntuaciones de la escala total como para las puntuaciones en las tres subescalas, y esto tanto en relación con la versión española como con las restantes versiones de la escala. El presente estudio incluye también las medias y desviaciones típicas relativas a cada uno de los 18 elementos de la escala; este tipo de datos aún no había sido publicado para la ASI-3. En línea con las publicaciones sobre la ASI (p.ej., Sandín et al., 2005), esperábamos encontrar diferencias significativas entre varones y mujeres en las puntuaciones de sensibilidad a la ansiedad (en general mayores puntuaciones en la mujer). Como puede observarse en la Tabla 3, las mujeres obtuvieron puntuaciones más elevadas en la escala total y en la subescala física. En nuestro estudio con la ASI también habíamos encontrado diferencias para la subescala cognitiva, si bien esta discrepancia podría deberse a errores de medida (i.e., grado limitado de fiabilidad y validez en esta subescala de la ASI). Por otra parte, en contra de lo esperado, los varones y mujeres sólo difieren en las puntuaciones a dos items de la dimensión somática y a un ítem de la dimensión social.

Cabría concluir diciendo que las diferencias entre hombres y mujeres en sensibilidad a la ansiedad son limitadas, si bien parece que las mujeres tienden a puntuar ligeramente más elevado que los varones en sensibilidad a la ansiedad general y en la faceta somática. Stewart et al. (1997), basándose en puntuaciones factoriales, al igual que nosotros sólo obtuvieron diferencias para la subescala somática. Puesto que no existen otros datos en la literatura sobre diferencias sexuales en las subescalas de sensibilidad a la ansiedad, futuros estudios deberán contrastar más específicamente esta cuestión. En principio, no obstante, si la dimensión social es un factor de vulnerabilidad más específico de la fobia social que de otros trastornos de ansiedad (Zinbarg et al., 1997), nuestros resultados son congruentes con el hecho de que las diferencias en fobias sociales entre varones y mujeres sean escasas y menores que en otros trastornos de ansiedad (Sandín, 1997, 1999), si bien en población infantojuvenil las niñas suelen informar mayores niveles de miedos, incluidos los miedos sociales (Pelechano, 1981, 1984; Sandín, Chorot, Valiente y Santed, 1998; Sandín, Valiente, Chorot, Santed y Sánchez-Arribas, 1999; Valiente, Sandín y Chorot, 2002; Valiente, Sandín, Chorot y Tabar, 2002, 2003).

La última cuestión que abordamos en la presente investigación concierne a la validez convergente y discriminante de la ASI-3. A este respecto, los resultados obtenidos son coherentes con nuestra hipótesis, ya que las variables de sensibilidad a la ansiedad correlacionaron de forma moderada y positiva con el afecto negativo, y de forma insignificante y/o negativa con el afecto positivo. Así mismo, en términos generales las correlaciones de sensibilidad a la ansiedad con los síntomas psicopatológicos siguen un patrón similar al informado en el estudio reciente de Zvolensky et al. (2003) sobre la ASI-R. Al igual que en este trabajo, nuestros resultados sugieren una relación positiva entre la sensibilidad a la ansiedad y las diferentes variables psicopatológicas, aunque en nuestro estudio el patrón se torna más discriminante tras controlar la influencia del afecto negativo a través de las correlaciones parciales.

Podemos observar que la ASI-3 total tiende a correlacionar de forma más elevada con las dimensiones psicopatológicas más relevantes al constructo de sensibilidad a la ansiedad, tales como la agorafobia, la obsesión-compulsión, la ansiedad y la somatización. Así mismo, merece la pena resaltar las correlaciones convergentes y discriminantes asociadas a las diferentes subescalas. Por ejemplo: (1) la depresión correlaciona de forma moderada con la ASI-3 cognitiva, pero muy débilmente con la ASI-3 física; (2) la correlación más elevada de la ASI-3 física corresponde a la agorafobia; (3) la agorafobia mantiene correlaciones destacadas con todas las variables de sensibilidad a la ansiedad; 
y (4) la hostilidad correlaciona de forma baja o irrelevante con las variables de sensibilidad a la ansiedad. Estos datos son consistentes con la evidencia de la literatura actual sobre las relaciones entre la sensibilidad a la ansiedad y la psicopatología (p.ej., Chorot, Sandín, Valiente, Santed, y Romero, 1997; Sandín et al., 1996, 2007; Taylor, 1999; Taylor et al., 2007).

Tal vez merezca un comentario aparte el patrón de correlaciones observado con la SA45 total. Como puede apreciarse, las correlaciones son apreciables incluso después de parcializar el efecto del afecto negativo, lo que sugiere que la sensibilidad a la ansiedad tal vez no es únicamente un factor de vulnerabilidad hacia los trastornos de ansiedad, sino también un potente factor de vulnerabilidad psicopatológica general, tal vez opuesto al concepto de resiliencia (Becoña, 2006).

Finalmente, las destacadas correlaciones entre la sensibilidad interpersonal y la sensibilidad a la ansiedad podrían explicarse por cierto solapamiento entre ambos tipos de variables, en especial entre la sensibilidad interpersonal y la ASI-3 social. Como ya hemos indicado en otro de nuestros trabajos (Sandín et al., 2007), la sensibilidad interpersonal definida en el SCL-90 es una variable mixta y relativamente confusa desde el punto de vista de su conceptuación psicopatológica.

En el presente estudio hemos proporcionado datos relevantes que apoyan de forma consistente la estructura factorial y la fiabilidad de la ASI3. Así mismo, nuestro trabajo aporta datos normativos de la escala, así como también evidencia preliminar sobre su validez convergente y discriminante. La ASI-3 es actualmente la escala de elección para la evaluación de la sensibilidad a la ansiedad, y posibilita la obtención válida y fiable de un índice general de sensibilidad a la ansiedad, así como también, índices de las tres dimensiones que han resultado ser relevantes (física, cognitiva y social). Los datos proporcionados, no obstante, se basan en población no clínica. Es necesario llevar a cabo nuevos estudios con población clínica para conocer las propiedades de la escala en este tipo de población, y en particular para probar su capacidad discriminante y predictiva sobre los trastornos de ansiedad y otros posibles trastornos psicológicos.

\section{REFERENCIAS}

Becoña, E. (2006). Resiliencia: Definición, características y utilidad del concepto. Revista de Psicopatología y Psicología Clínica, 11, 125-146.

Bentler, P.M. (2005). EQS Structural equations program manual. Encino, CA: Multivariate Software, Inc.

Brown, M., Smits, J.A.J., Powers, M.B., \& Telch, M.J. (2003). Differential sensitivity of the three ASI factors in predicting panic disorder patients' subjective and behavioural response to hyperventilation challenge. Journal of Anxiety Disorders, 17, 583-591.

Cabrera, P., Urrutia, B., Vera, V., Alvarado, M., y VeraVillaroel, P. (2005). Ansiedad y depresión en niños diagnosticados de cáncer. Revista de Psicopatología y Psicología Clínica, 10, 103-114.

Cintrón, J.A., Carter, M.M., Suchday, S., Sbrocco, T., \& Gray, J. (2005). Factor structure and construct validity of the Anxiety Sensitivity Index among island Puerto Rico. Journal of Anxiety Disorders, 19, 51-68.

Chorot, P., Sandín, B., Valiente, R.M., Santed, M.A., y Romero, M. (1997). Actitud hacia la enfermedad, ansiedad y sintomatología somática en pacientes con trastorno de pánico e hipocondría. Revista de Psicopatología y Psicología Clínica, 2, 123-136.

Davison, M.K., Bershadsky, B., Bieber, J., Silversmith, D., Maruish, M.E., \& Kane, R.L. (1997). Development of a brief, multidimensional, self-report instrument for treatment outcomes assessment in psychiatric settings: Preliminary findings. Assessment, 4, 259-276.

Deacon, B.J., Abramowitz, J.A., Woods, C.M., \& Tolin, D.F. (2003). The Anxiety Sensitivity Index-Revised: Psychometric properties and factor structure in two nonclinical samples. Behaviour Research and Therapy, 41, 1427-1449.

Gimeno, F., y Ezquerro, M. (2006). Intervención psicológica en un caso de evitación interoceptiva en el deporte. Revista de Psicopatología y Psicología Clínica, 11, 99-106.

Nuevo, R. (2005). Sensibilidad y especificidad del Cuestionario de Preocupación y Ansiedad para la detección del trastorno de ansiedad generalizada. Revista de Psicopatología y Psicología Clínica, 10, 136-142.

Pelechano, V. (1981). Miedos infantiles y terapia familiarnatural. Valencia: Alfaplús.

Pelechano, V. (1984). Programas de intervención psicológica en la infancia. Valencia: Alfaplús.

Reiss, S. (1991). Expectancy model of fear, anxiety, and panic. Clinical Psychology Review, 11, 141-153.

Reiss, S., Peterson, R.A., Gursky, D.M., \& McNally, R.J. (1986). Anxiety sensitivity, anxiety frequency, and the prediction of fearfulness. Behaviour Research and Therapy, $24,1-8$. 
Sandín, B. (2005a). Evitación interoceptiva: Nuevo constructo en el campo de los trastornos de ansiedad. Revista de Psicopatología y Psicología Clínica, 10, 103114.

Sandín, B. (2005b). El síndrome de fatiga crónica: Características psicológicas y terapia cognitivo-conductual. Revista de Psicopatología y Psicología Clínica, 10, 8594.

Sandín, B. (1997). Ansiedad, miedos y fobias en niños y adolescentes. Madrid: Dykinson.

Sandín, B. (1999). Las fobias específicas. Madrid: Klinik.

Sandín, B., Chorot, P., \& McNally, R.J. (1996). Validation of the Spanish version of the Anxiety Sensitivity Index in a clinical sample. Behaviour Research and Therapy, 34, 283-209.

Sandín, B., Chorot, P., Lostao, L., Joiner, T.E., Santed, M.A., y Valiente, R.M. (1999). Escalas PANAS de afecto positivo y negativo: Validación factorial y convergencia transcultural. Psicothema, 11, 37-51.

Sandín, B., Chorot, P., Santed, M.A., y Valiente, R.M. (2002a). Análisis factorial confirmatorio del Índice de Sensibilidad a la Ansiedad para Niños. Psicothema, 14, 333-339.

Sandín, B., Chorot, P., Valiente, R.M., y Santed, M.A. (1998). Frecuencia e intensidad de los miedos en los niños: Datos normativos. Revista de Psicopatología y Psicología Clínica, 3, 15-25.

Sandín, B., Chorot, P., Valiente, R.M., y Santed, M.A. (2002b). Relación entre la sensibilidad a la ansiedad y el nivel de miedos en niños. Psicología Conductual, 10, 107-120.

Sandín, B., Chorot, P., Valiente, R.M., Santed, M.A., y Lostao, L. (2004a). Dimensiones de sensibilidad a la ansiedad: Evidencia confirmatoria de la estructura jerárquica. Revista de Psicopatología y Psicología Clinica, 9, 19-33.

Sandín, B., Rodero, B., Santed, M.A., y García-Campayo, J. (2006). Sucesos vitales estresantes y trastorno de pánico: Relación con la ocurrencia del trastorno de pánico, la gravedad clínica y la agorafobia. Revista de Psicopatología y Psicología Clínica, 11, 179-190.

Sandín, B., Valiente, R.M., Chorot, P., y Santed, M.A (2005). Propiedades psicométricas del Índice de Sensibilidad a la Ansiedad. Psicothema, 17, 478-483.

Sandín, B., Valiente, R.M., Chorot, P., y Santed, M.A. (2004b). Escala de Sensibilidad a la Ansiedad-Revisada (ASI-R). Ponencia invitada de Symposium. VII European Conference on Psychological Assessment. Benalmádena Costa (Málaga), 1-4 de abril, p. 77 (Libro de Actas).

Sandín, B., Valiente, R.M., Chorot, P., Santed, M.A. (2007). SA-45: Forma abreviada del SCL-90. Psicothema. Enviado para publicación.
Sandín, B., Valiente, R.M., Chorot, P., Santed, M.A., y Sánchez-Arribas, C. (1999). Escala de Ansiedad Social para Niños-Revisada (SASC-R): Fiabilidad, validez y datos normativos. Análisis y Modificación de Conducta, 25, 827-847.

Stewart, S.H., Taylor, S., \& Baker, J.M. (1997). Gender differences in dimensions of anxiety sensitivity. Journal of Anxiety Disorders, 11, 179-200.

Taylor, S. (Ed.) (1999). Anxiety sensitivity: Theory, research, and treatment of the fear of anxiety. Mahwah, $\mathrm{NJ}$ : LEA.

Taylor, S., Koch, W.J., Woody, S., \& McLean, P. (1996). Anxiety sensitivity and depression: How are they related? Journal of Abnormal Psychology, 105, 474-479.

Taylor, S., y Cox, B.J. (1998). An expanded Anxiety Sensitivity Index: Evidence for a Hierarchic structure in a clinical sample. Journal of Anxiety Disorders, 12, 463483.

Taylor, S., Zvolensky, M.J., Cox, B.J., Deacon, B., Heimberg, R.G., Ledley, D.R., Abramowith, J.S., Holaway, R.M., Sandín, B., Stewart, S.H., Coles, M., Eng, W., Daly, E.S., Arrindell, W.A., Bouvard, M., \& Jurado Cardenas, S. (2007). Robust dimensions of anxiety sensitivity: Development and initial validation of the Anxiety Sensitivity Index-3 (ASI-3). Psychological Assessment, 19, 176-188.

Valiente, R.M., Sandín, B., y Chorot, P. (2002). Miedos comunes en niños y adolescentes: Relación con la sensibilidad a la ansiedad, el rasgo de ansiedad, la afectividad negativa y la depresión. Revista de Psicopatología y Psicología Clínica, 7, 61-70.

Valiente, R.M., Sandín, B., Chorot, P., y Tabar, A. (2002). Diferencias sexuales en la prevalencia e intensidad de los miedos durante la infancia y la adolescencia: datos basados en el FSSC-R. Revista de Psicopatología y Psicología Clínica, 7, 103-113.

Valiente, R.M., Sandín, B., Chorot, P., y Tabar, A. (2003). Diferencias según la edad en la prevalencia e intensidad de los miedos durante la infancia y la adolescencia: datos basados en el FSSC-R. Psicothema, 15, 414419.

Watson, D., Clark, L.A., \& Tellegen, A. (1988). Development and validation of brief measures of positive and negative affect: The PANAS scales. Journal of Personality and Social Psychology, 54, 1063-1070.

Zinbarg, R.E., Barlow, D.H., \& Brown, T.A. (1997). Hierarchical structure and general factor saturation of the Anxiety Sensitivity Index: Evidence and implications. Psychological Assessment, 9, 277-284.

Zvolensky, M.J., Arrindell, W.A., Taylor, S., Bouvard, M., Cox, B.J., Stewart, S.H., Sandín, B., Jurado Cardenas, S., \& Eifert, G.H. (2003). Anxiety sensitivity in six countries. Behaviour Research and Therapy, 41, 841859 . 


\title{
Anexo I. Anxiety Sensitivity Index-3 (ASI-3)
}

\begin{abstract}
ASI-3
Fecha

Edad

Sexo:

Mujer

Varón

Conteste rodeando con un círculo el número $(0,1,2,3,4)$ que mejor refleje su experiencia con lo que se indica en cada uno de los enunciados. Si algo de lo que se dice no lo ha sentido o experimentado nunca (p.ej., desmayarse en público), conteste como usted crea que se sentiría si realmente le hubiera ocurrido.
\end{abstract}

Responda a todos los enunciados teniendo en cuenta la siguiente valoración:

$\begin{array}{cccccc}0 & 1 & 2 & 3 & 4 \\ \qquad & \mid & \text { Un poco } & \text { Bastante } & \text { Mucho } & \text { Muchísimo }\end{array}$

Casi nada

1. Para mí es importante no dar la impresión de estar nervioso/a

$\begin{array}{lllll}0 & 1 & 2 & 3 & 4\end{array}$

2. Cuando no puedo mantener mi mente concentrada en una tarea, siento la preocupación de que podría estar volviéndome loco/a

\begin{tabular}{lllll}
0 & 1 & 2 & 3 & 4 \\
\hline 0 & 1 & 2 & 3 & 4 \\
0 & 1 & 2 & 3 & 4 \\
0 & 1 & 2 & 3 & 4 \\
\hline 0 & 1 & 2 & 3 & 4 \\
0 & 1 & 2 & 3 & 4 \\
\hline 0 & 1 & 2 & 3 & 4 \\
0 & 1 & 2 & 3 & 4 \\
\hline 0 & 1 & 2 & 3 & 4 \\
\hline 0 & 1 & 2 & 3 & 4
\end{tabular}

3. Me asusto cuando mi corazón late de forma rápida

4. Cuando siento malestar en el estómago, me preocupa estar seriamente enfermo/a

5. Me asusto cuando soy incapaz de mantener mi mente concentrada en una tarea

6. Cuando tiemblo en presencia de otras personas, me da miedo lo que puedan pensar de mí

7. Cuando siento opresión en el pecho, me asusta no poder respirar bien

8. Cuando siento dolor en el pecho, me preocupa que vaya a darme un ataque cardíaco

9. Me preocupa que otras personas noten mi ansiedad

10. Cuando tengo la sensación de que las cosas no son reales, me preocupa que pueda estar mentalmente enfermo/a

11. Tengo miedo a sonrojarme delante de la gente

$\begin{array}{lllll}0 & 1 & 2 & 3 & 4\end{array}$

12. Cuando noto que mi corazón da un salto o late de forma irregular, me preocupa que algo grave me esté ocurriendo

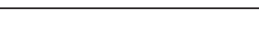

13. Cuando comienzo a sudar en una situación social, me da miedo que la gente piense negativamente de mí

14. Cuando mis pensamientos parecen acelerarse, me preocupa que pueda volverme loco/a

15. Cuando siento opresión en la garganta, me preocupa que pueda atragantarme y morir

16. Cuando me resulta difícil pensar con claridad, me preocupa que me esté ocurriendo algo grave

17. Pienso que me resultaría horrible si me desmayase en público

$\begin{array}{lllll}0 & 1 & 2 & 3 & 4\end{array}$

18. Cuando mi mente se queda en blanco, me preocupa que me esté ocurriendo algo terriblemente malo

$\begin{array}{lllll}0 & 1 & 2 & 3 & 4\end{array}$

$\begin{array}{lllll}0 & 1 & 2 & 3 & 4\end{array}$

$\begin{array}{lllll}0 & 1 & 2 & 3 & 4\end{array}$

$\begin{array}{lllll}0 & 1 & 2 & 3 & 4\end{array}$

$\begin{array}{lllll}0 & 1 & 2 & 3 & 4\end{array}$

Nota. Clave de corrección: Física = suma de los Items 3, 4, 7, 8, 12, 15. Cognitiva = suma de los Items 2, 5, 10, 14, $16,18$. Social $=$ suma de los Items 1, 6, 9, 11, 13, 17 .

(C) Versión española B. Sandín ${ }^{1}$, P. Chorot ${ }^{1}$, y R.J. McNally ${ }^{2} ;{ }^{1} \mathrm{UNED}$, Madrid y ${ }^{2}$ Harvard University, USA 